\title{
Effects of Clearance on Deployment of Solar Panels on Spacecraft System
}

\author{
By Yang ZHAO and ZhengFeng BAI \\ Department of Astronautics Engineering, Harbin Institute of Technology, Harbin, People's Republic of China
}

(Received November 29th, 2009)

\begin{abstract}
This paper establishes a dynamics model for deploying solar panels with clearances by using a practical method and provides a useful way to identify the effects of clearances on spacecraft systems. Considering the clearance of joints, a contact dynamic model is established using the nonlinear spring-damp model and the friction effect is considered by using the Coulomb friction model. Based on the model, numeric simulation of the deployment of a single panel with clearance on a spacecraft is presented. The effects of the clearance on attitude motion of the spacecraft as well as the deployment of a solar panel with joint clearance are analyzed. The simulation results predict the effects of clearance on the attitude motion of a spacecraft system and the deployment dynamics of solar panel preferably. It is useful for engineering design of a spacecraft control system and ground text. This work provides a valuable method for improving the dynamics of spacecraft systems.
\end{abstract}

Key Words: Solar Panel, Clearance, Dynamics Model, Contact, Attitude Motion

\section{Introduction}

Modern spacecrafts, which usually have large deployment solar panels, are becoming larger and larger with the rapid development of space technology. Considering the launch vehicle size and large payloads, solar panels usually are folded during launch. There are freed and deployed by drive springs when the spacecraft and launch vehicle separated. Therefore deployment of solar panels is an important process in achieving stable orbit.

Deployment of solar panels usually involves various joints. However, joint clearances are inevitable due to manufacturing tolerances, imperfections, wear and material deformation. Clearances can cause contact and impact between the jointed solar panels during deployment. Hence, internal forces in joints cause severe vibration, which increase the dynamic stress of solar panels. Moreover, they affect the attitude motion of the spacecraft and deployment accuracy, which affect spacecraft performance.

Deployment of solar panels has been studied extensively. ${ }^{1-4)}$ However, in these studies, joints are treated as perfect, i.e. no clearance in joints. This paper focuses on deployment of solar panels using joints with clearances. A contact dynamics model is established using the nonlinear spring-damp model and the friction effect is considered using a Coulomb friction model. The effects of clearance on the attitude motion of spacecraft and dynamic response to deployment of solar panels are analyzed.

\section{Model for Revolute Clearance Joint}

\subsection{Definition of clearance}

All joints have clearance to allow relative motion of connected bodies, and permit assembly of the spacecraft

(C) 2011 The Japan Society for Aeronautical and Space Sciences system. It is known that the performance of a spacecraft is degraded by the presence of clearance due to impact force, which contributes to bearing failure due to shock loading, reduced life, due to material fatigue, causing energy dissipation and unwanted vibration responses.

In general, a clearance joint can be included in a spacecraft system much like a revolute joint. The classical approach, known as zero-clearance approach, treats the connecting points of two bodies linked by a revolute joint as coincident. Introduction of clearance in a joint separates these two points. Figure 1 shows a revolute joint with clearance. The difference in radius between the bearing and journal defines the size of the radial clearance. ${ }^{5)}$ Although, a revolute joint with clearance does not constrain any degree of freedom from the mechanical system like an ideal joint, it imposes some kinematic restrictions, limiting the journal to move within the bearing. Thus, when there is clearance in a revolute joint, the two kinematic constraints are removed and two degrees of freedom are introduced. The dynamics of the joint are then controlled by forces working on the journal and bearing. Thus, while a perfect revolute joint in a mechanical system imposes kinematic constraints, a revolute clearance joint leads to force constraints. When there is contact between the journal and bearing, a contact force is applied perpendicular to the plane of collision.

Deployment of solar panels on spacecraft with joints and clearance always includes contact/impact. The key point of the dynamics model for solar panels with clearance is to incorporate a clearance model and a dynamics model, requiring accurate definition of the contact/impact process in joint clearance.

The difference in radius between the bearing and journal defines the size of radial clearance, so clearance is defined as follows: 


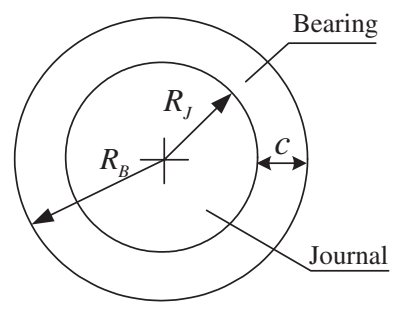

Fig. 1. Diagram of revolute joint with clearance.

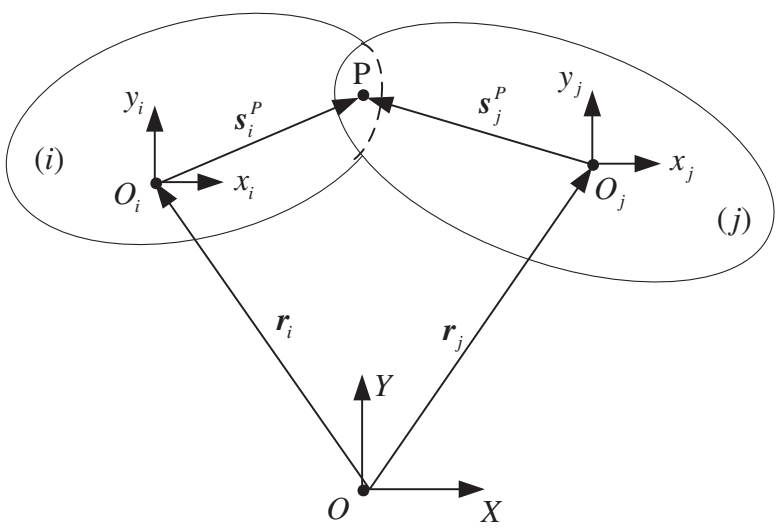

Fig. 2. Planar revolution joint connecting bodies $i$ and $j$.

$$
c=R_{\mathrm{B}}-R_{\mathrm{J}}
$$

where, $R_{\mathrm{B}}$ and $R_{\mathrm{J}}$ are the radius of the bearing and journal, respectively.

\subsection{Contact model}

To evaluate contact forces between the bearing and journal in a revolute joint with clearance, special attention must be given to the numerical description of the contact model. ${ }^{6}$ Information on the impact velocity, material properties of the colliding bodies and geometry characteristics of the surfaces in contact must be included in a force contact model. These characteristics are observed with continuous contact force, in which deformation and contact force are considered as continuous functions.

The simplest contact force model is spring-damp model, modeled by a parallel spring-damp element. The spring represents the elasticity of the contacting bodies, and the damper describes the loss of kinetic energy during impact. The contact force depends on the stiffness and damping properties of the spring. Although the spring-damp model is an approximate method, it can simulate contact/impact characteristics in clearances perfectly, and the simulation results are accurate when reasonable stiffness and damping parameters are selected.

The formula for the contact model, considering clearance using a spring-damp model is generally

$$
F_{\mathrm{n}}=F_{\mathrm{k}}+F_{\mathrm{d}}=K_{\mathrm{n}} \delta^{m}+c(\delta) \dot{\delta}
$$

where, $F_{\mathrm{n}}$ is the normal contact force, $F_{\mathrm{d}}$ is the damp force, $K_{\mathrm{n}}$ is the generalized contact stiffness, $\delta$ is the penetration depth, $c(\delta)$ is the damp factor related with $\delta$, and $m$ is an exponent.
The best-known contact force law between two spheres of isotropic material is based on the pioneering work of Hertz. The normal contact/impact force is defined by the Hertz contact theory. The nonlinear normal contact/impact force $F_{\mathrm{k}}$, know as the Hertz force-displacement law, is evaluated as a function of indentation between two colliding bodies, as follows:

$$
F_{\mathrm{k}}=K_{\mathrm{n}} \delta^{1.5}
$$

where, $\delta$ is the penetration depth. The exponent $m$ is generally set to 1.5 .

To overcome the limitations of the linear damping model and to satisfy the boundary conditions, the damping force $F_{\mathrm{d}}$ is defined, using the non-linear damping model, as follows:

$$
F_{\mathrm{d}}=c(\delta) \dot{\delta}
$$

where, $c(\delta)$ is the damping factor related to $\delta$. In this paper, $c(\delta)$ is defined as

$$
c(\delta)=0.75\left(1-e^{2}\right) K_{\mathrm{n}} \delta^{3 / 2} / v_{0}
$$

where, $e$ is the coefficient of restitution, and $v_{0}$ is the initial impact velocity.

The tangential friction force, known as the Coulomb, is evaluated as follows:

$$
\begin{aligned}
& F_{\mathrm{t}}=-\mu_{\mathrm{d}} F_{\mathrm{n}} \operatorname{sgn}(v) \\
& v(q, \dot{q}, t) \neq 0
\end{aligned}
$$

where, $\operatorname{sgn}(v)$ is a sign function, $v(q, \dot{q}, t) \neq 0$ is the relative slip velocity between the contact points of the bodies, and $\mu_{\mathrm{d}}$ is the coefficient of sliding friction.

\subsection{Dynamics equation of spacecraft with clearance \\ 2.3.1. Constraint equation}

A kinematic joint imposes some conditions on the relative motion between the adjacent bodies comprising the joint. When these conditions are expressed in analytical form, they are called constraint equations. A constraint is any condition that reduces the number of degrees of freedom in a system. ${ }^{7)}$

The revolute joint is a bearing and journal type of joint that constrains the relative translation between the two bodies $i$ and $j$, allowing only relative rotations, as shown in Fig. 2. The kinematic conditions for the revolute joint require that two separate points, each one belonging to a different body, share the same position in space all the time. This means that the global position of a point $\mathrm{P}$ in body $i$ is coincident with the global position of a point $\mathrm{P}$ in body $j$. Such a condition is expressed by two algebraic equations obtained from the following vector loop equation,

$$
\boldsymbol{r}_{i}+\boldsymbol{s}_{i}^{\mathrm{P}}-\boldsymbol{r}_{i}-\boldsymbol{s}_{j}^{\mathrm{P}}=0
$$

which is re-written as,

$$
\Phi=\boldsymbol{r}_{i}+\boldsymbol{A}_{i} \boldsymbol{s}_{i}^{\mathrm{P}}-\boldsymbol{r}_{j}-\boldsymbol{A}_{j} \boldsymbol{s}_{j}^{\mathrm{P}}=0
$$

Thus, there is only one relative degree of freedom (DOF) between two bodies connected by a planar revolute joint. 


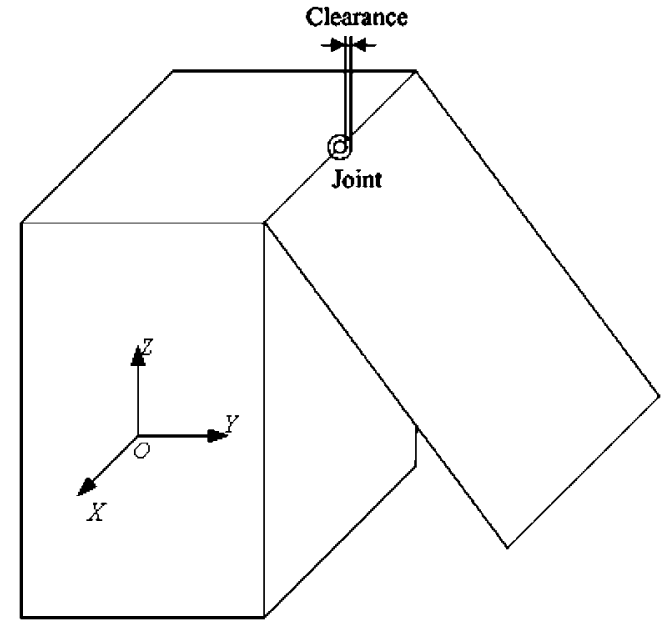

Fig. 3. Diagram of spacecraft system with clearance joint.

Table 1. Mass parameters of spacecraft system.

\begin{tabular}{lcl}
\hline & Body & Solar panel \\
\hline Mass $(\mathrm{kg})$ & 768.0 & 7.68 \\
$J_{x}\left(\mathrm{~kg} \cdot \mathrm{m}^{2}\right)$ & 104.96 & 1.6137 \\
$J_{y}\left(\mathrm{~kg} \cdot \mathrm{m}^{2}\right)$ & 81.92 & 0.4156 \\
$J_{z}\left(\mathrm{~kg} \cdot \mathrm{m}^{2}\right)$ & 104.96 & 2.0293 \\
$J_{x y}\left(\mathrm{~kg} \cdot \mathrm{m}^{2}\right)$ & 0 & 0 \\
$J_{y z}\left(\mathrm{~kg} \cdot \mathrm{m}^{2}\right)$ & 0 & 0 \\
$J_{z x}\left(\mathrm{~kg} \cdot \mathrm{m}^{2}\right)$ & 0 & 0 \\
\hline
\end{tabular}

For a constrained multi-body system, the kinematical joints are described by a set of holonomic algebraic constraints and $\Phi$ can be written in compact form as ${ }^{7,8)}$

$$
\Phi(\boldsymbol{q}, t)=0
$$

where, $q$ is the vector of generalized coordinates, and $t$ is the time variable, in general associated with the driving elements.

\subsubsection{Dynamic equation of spacecraft with clearance}

A dynamics model for a spacecraft system is established based on the clearance model. The joint clearance leads to two different motion phases for connected bodies: the bodies move free in the clearance and the bodies contact and interact. So a spacecraft system with clearances between bodies is a variable topology system.

The variable topology system for deployment of solar panels on a spacecraft system is solved using the dynamic segmentation modeling method. The dynamic equation is obtained by the Lagrange multiplier method and the dynamic equation is formulated as Eq. (10) and Eq. (11). ${ }^{9,10)}$ For details refer to references 7,9 ) and 10).

In the free-motion phase, the dynamic equation is

$$
\begin{aligned}
& M \ddot{q}+C \dot{q}+K q+\Phi_{\mathrm{q}}{ }^{\mathrm{T}} \lambda=f \\
& \Phi(q, t)=0
\end{aligned}
$$

where, $q$ is the generalized coordinate column matrix, and $M, C$ and $K$ are the generalized mass matrix, generalized damping matrix, and generalized stiffness matrix, respectively. $\Phi_{\mathrm{q}}$ is the Jacobin matrix of constraint equation. $f$ is

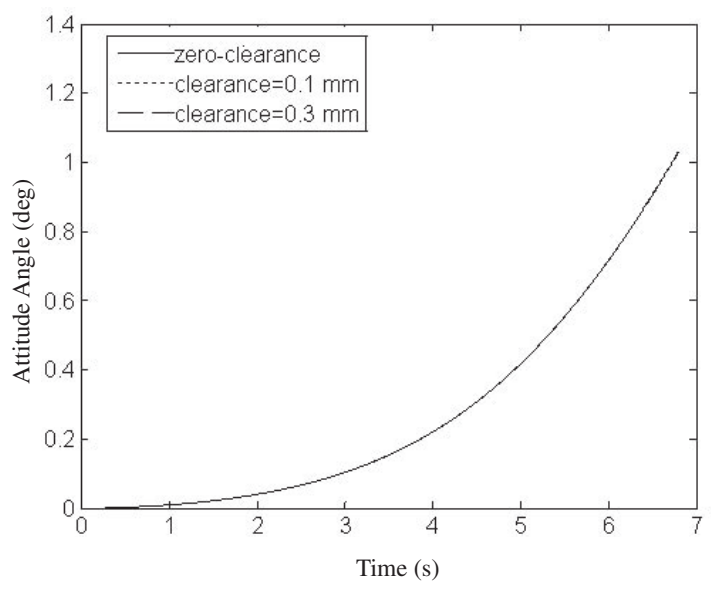

(a)

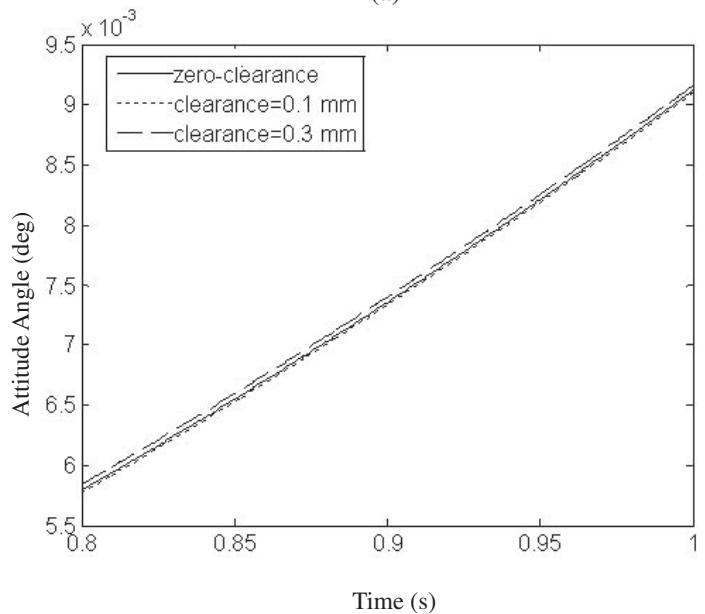

(b)

Fig. 4. Attitude angle of spacecraft ((a) whole process and (b) partial enlarged).

the generalized force matrix. $\lambda$ is the Lagrange multiplier column matrix.

In the contact phase, the bodies contact and interact, so there are contact forces in the clearances. The dynamic equation is

$$
\begin{aligned}
& M \ddot{q}+C \dot{q}+K q+\Phi_{\mathrm{q}}{ }^{\mathrm{T}} \lambda=f+F_{\mathrm{C}} \\
& \Phi(q, t)=0
\end{aligned}
$$

where, $F_{\mathrm{C}}$ is the contact force relative to $q$, which contains both the normal contact force, $F_{\mathrm{n}}$, and tangential friction force, $F_{\mathrm{t}}$.

\section{Dynamics Simulation and Results}

\subsection{Properties of spacecraft system}

A single-panel spacecraft system is used to study the effects of clearance on attitude motion. Figure 3 shows the configuration, which consists of two bodies that represent the center body and solar panel, as one deployment mechanism. There is a revolute clearance joint between the center body and solar panel. The mass and inertia properties of the system are shown in Table 1.

The dynamics simulation, analyzed three simulations: zero-clearance; 0.1-mm clearance, and 0.3-mm clearance, 


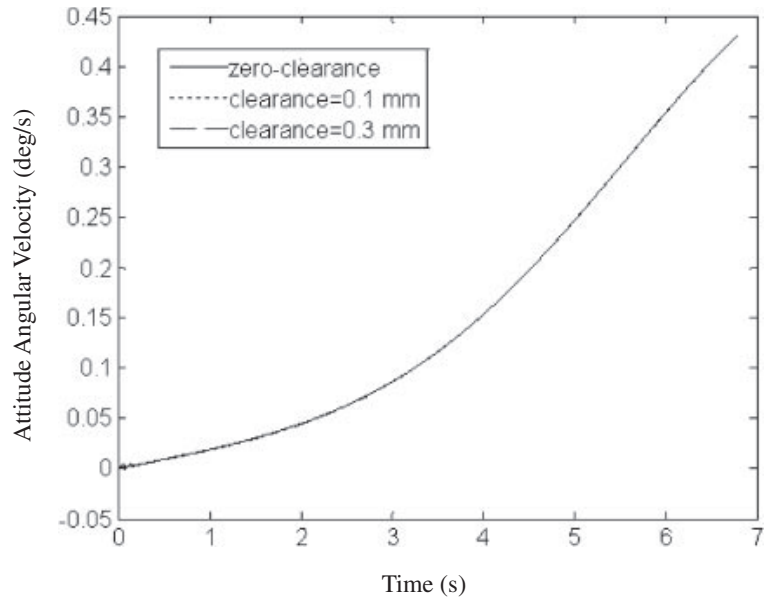

(a)

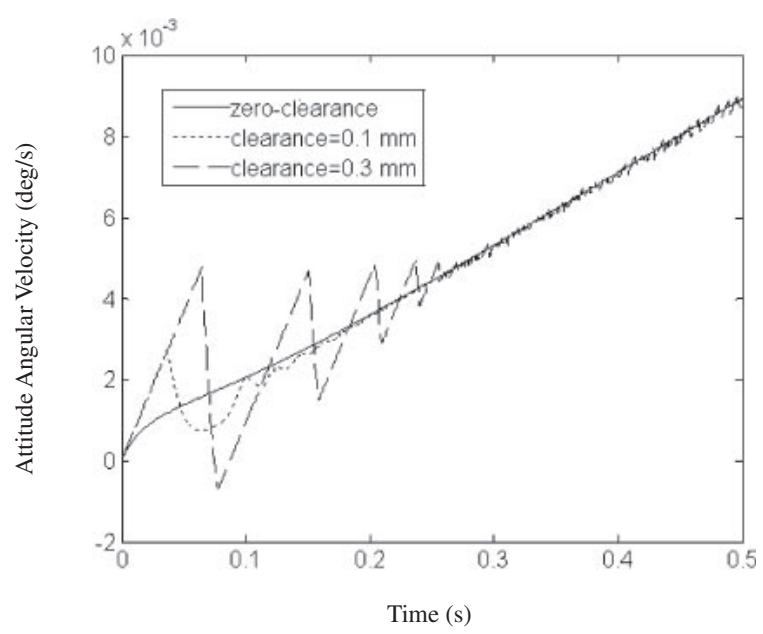

(b)

Fig. 5. Attitude angular velocity of spacecraft ((a) whole process and (b) partial enlarged).

where contact stiffness $K_{\mathrm{n}}=1.5 \times 10^{5} \mathrm{~N} / \mathrm{m}^{2}$ and coefficient of sliding friction $\mu_{\mathrm{d}}=0.1$.

Deployment is driven by the joint torsion spring represented as follows:

$$
T=T_{0}-k \theta
$$

where, $T_{0}$ is the initial moment of torsion, $k$ is the stiffness of the torsion spring, and $\theta$ is the deployment angle of the solar panel. The parameters of the torsion spring are $T_{0}=0.135 \mathrm{Nm}$ and $k=0.0015 \mathrm{Nm} / \mathrm{deg}$.

\subsection{Simulation results}

Figures 4 and 5 show the attitude motion of the spacecraft system; Fig. 6 and Fig. 7 show the deployment responses of the solar panel.

Figure 4 shows the attitude angles of the spacecraft system are similar with clearances of the three sizes. The difference between the three clearances is small, indicating that the effect of clearance on attitude angles is slight.

Figure 5 shows the clear effect of clearance on attitude angular velocities. The attitude angular velocities with clearances contain large vibrations at the start of deployment of the solar panel and the attitude angular velocities

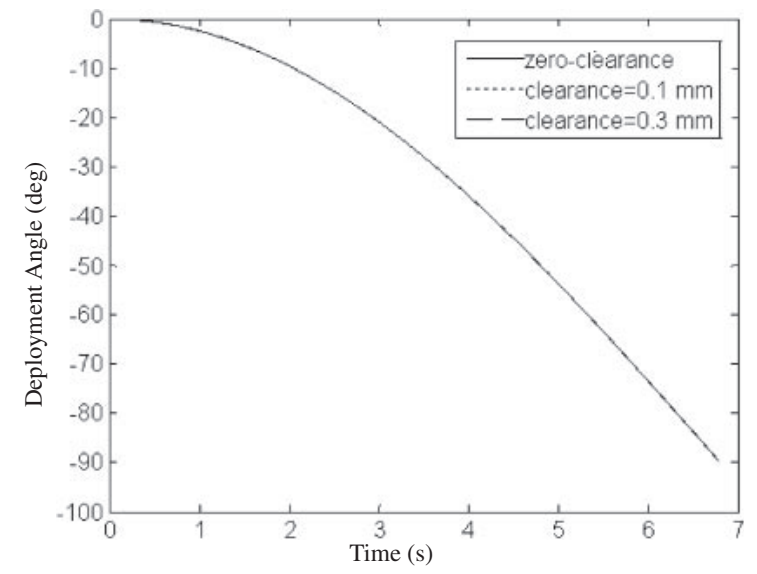

(a)

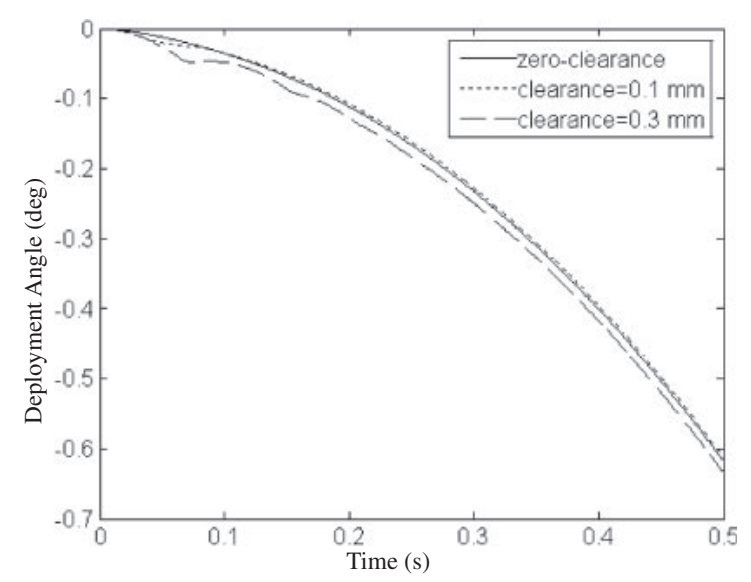

(b)

Fig. 6. Deployment angle of solar panel ((a) whole deployment process and (b) partial enlarged).

are obviously vibrating around the attitude angular velocity with zero-clearance. Moreover, the attitude angular velocity responses show that bigger clearance induces higher frequency vibration and larger amplitude.

The results clarify that angular velocity is sensitive to clearance, and the effects of clearance on attitude angular velocities are severe than the effects of clearance on attitude angles.

Figure 6 shows the deployment angles of solar panel are similar with clearance of three sizes. The differences in the deployment angles between the three cases is slight, indicating that the effect of clearance on deployment angles of the solar panel is slight.

Figure 7 shows the clear effect of clearance on deployment angular velocities of the solar panels. The deployment angular velocities with clearances contain large vibrations at the start of panel deployment. The deployment angular velocities are obviously vibrating compared to the deployment angular velocity of the panel with zero-clearance. Moreover, the deployment angular velocity responses show that bigger clearance leads to higher frequency vibration and larger vibration amplitude for the angular velocity.

The results show that angular velocity is sensitive to clearance and the effects of clearance on deployment angu- 


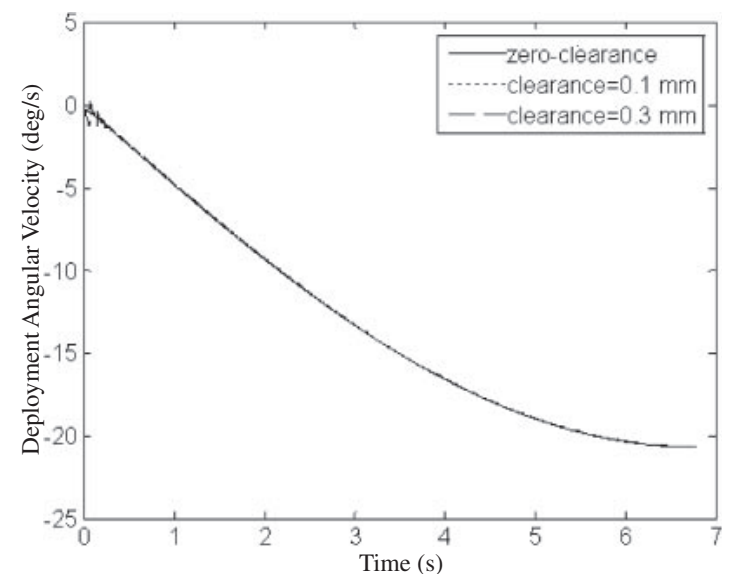

(a)

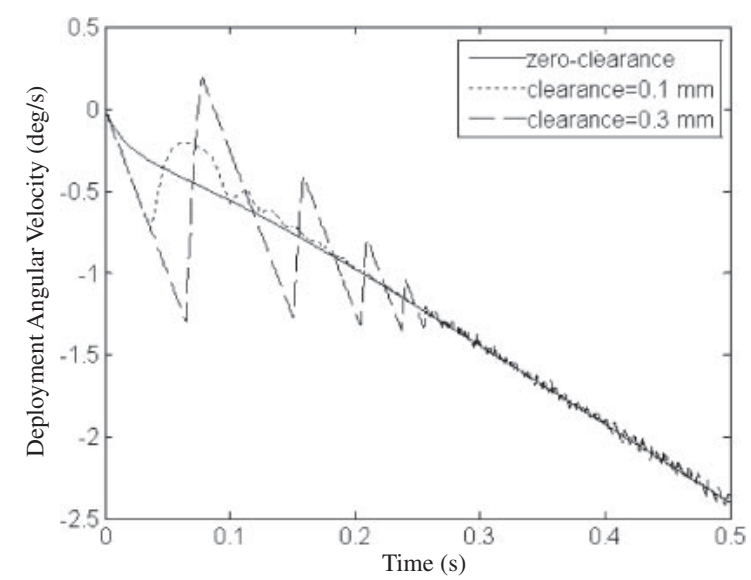

(b)

Fig. 7. Deployment angular velocity of solar panel ((a) whole deployment process and (b) partial enlarged).

lar velocities of solar panels are more obvious than the effects of clearance on deployment angle.

\section{Conclusion}

(1) The effects of clearance both on the attitude angles of the spacecraft system and the deployment angle of solar panels are slight. However, the effects of clearance on both attitude angular velocities and deployment angular velocities of solar panels are evident. The angular velocities with clearances contain large vibrations at the start of solar panel deployment. The angular velocities are obviously vibrating compared to the angular velocity of the spacecraft system with zero-clearance, indicating that angular velocity is sensitive to clearance.

(2) Bigger clearance causes higher-frequency vibrations and larger-amplitude vibration of the attitude angular velocity of the spacecraft and solar panel deployment angular velocity.

The simulation results can predict the effects of clearance on spacecraft attitude and solar panel deployment dynamics. This work provides a valuable method for improving the dynamics of spacecraft systems.

\section{References}

1) Wie, B., Furumoto, N., Banerjee, A. K. and Barba, P.M.: Modeling and Simulation of Spacecraft Solar Array Deployment, J. Guidance, 9, 5 (1986), pp. 593-598.

2) Oskar, W. and Simon, W.: Simulation of Deployment of a Flexible Solar Array, Multibody Syst. Dyn., 7 (2002), pp. 101-125.

3) Jorgensen, J. R., Louis, E. L., Hinkle, J. D. and Silver, M. J.: Dynamics of an Elastically Deployable Solar Array: Ground Test Model Validation Multibody System Dynamics, AIAA/ASME/ASCE/AHS/ASC Structures, Structural Dynamics \& Materials Conference, Austin, Texas, Vol. 4, 2005, pp. 18-21.

4) Bai, Z. F., Zhao, Y. and Tian, H.: Dynamics Simulation of Deployment of Solar Panels in Fault Modes, J. System Simulation, 19, 13 (2007), pp. 3067-3072.

5) Flores, P., Ambrosio, J., Claro, H. C. P., Lankarani, H. M. and Koshy, C. S.: A Study on Dynamics of Mechanical Systems Including Joints with Clearance and Lubrication, Mech. Mach. Theory, 41 (2006), pp. 247-261.

6) Garcia Oeden, J. C.: Analysis of Clearance in Multibody System, Multibody Syst. Dyn., 13 (2005), pp. 401-420.

7) Nikravesh, P. E.: Computer-Aided Analysis of Mechanical Systems, Prentice-Hall, Englewood Cliffs, NJ, 1988.

8) Flores, P. and Ambrosio, J.: Revolution Joints with Clearance in Multibody System, Comput. Struct., 82(2004), pp. 1359-1369.

9) Dubowsky, S., Deck, J. F. and Costello, H.: The Dynamic Modeling of Flexible Spatial Machine Systems with Clearance Connections, J. Mech. Transmissions Automation Design, 109 (1987), pp. 87-94.

10) Yan, S. Z., Chen, L. M. and Ji, H. L.: Dynamic Modeling of Multibody System with Clearance Joints, J. Vib. Engineering, 16 (2003), pp. 290294. 